\title{
1HNMR-based metabolomic profile of rats with experimental acute pancreatitis
}

\author{
Juan $\mathrm{Li}^{1 \dagger}$, Xian-lin Zhao ${ }^{1 \dagger}$, Yi-xia Liu' ${ }^{1,2}$, Xiao-hang Peng ${ }^{1}$, Shi-feng Zhu', Hui Guo', Yi-Ling Liu', Mei-hua Wan \\ and Wen-fu Tang ${ }^{1 *}$
}

\begin{abstract}
Background: Acute pancreatitis (AP) is a common inflammatory disease of the pancreas accompanied by serious metabolic disturbances. Nevertheless, the specific metabolic process of this disease is still unclear. Characterization of the metabolome may help identify biomarkers for AP. To identify potential biomarkers, this study therefore investigated the $1 \mathrm{H}$-nuclear magnetic resonance (NMR)-based metabolomic profile of AP.
\end{abstract}

Methods: Fourteen male adult Sprague-Dawley rats were randomized into two groups: the AP group, in which AP was induced by retrograde ductal infusion of 3.5\% sodium taurocholate; and the sham operation group (SO), in which rats were infused with $0.9 \%$ saline. Blood samples were obtained 12 hours later and a $600 \mathrm{MHz}$ superconducting NMR spectrometer was used to detect plasma metabolites. Principal components analysis (PCA) and partial least squares-discriminant analysis after orthogonal signal correction (OSC-PLS-DA) were used to analyze both longitudinal Eddy-delay (LED) and Carr-Purcell-Meiboom-Gill (CPMG) spectra.

Results: Differences in plasma metabolites between the two groups were detected by PCA and PLS-DA of $1 \mathrm{HNMR}$ spectra. Compared with the SO group, plasma levels of lactate $(\delta 1.3,1.34,4.1)$, valine $(\delta 0.98,1.02)$, succinic acid ( $\delta$ 2.38), 3-hydroxybutyric acid (3-HB, $\delta 1.18)$, high density lipoprotein (HDL, $\delta 0.8)$, and unsaturated fatty acid (UFA, $\delta 2.78,5.3)$ were elevated in the AP group, while levels of glycerol $(\delta 3.58,3.66)$, choline $(\delta 3.22)$, trimethylamine oxide (TMAO, $\delta 3.26)$, glucose $(\delta 3-4)$, glycine $(\delta 3.54)$, very low density lipoprotein $(V L D L, \delta 1.34)$ and phosphatidylcholine (Ptd, $\delta$ 2.78) were decreased.

Conclusions: AP has a characteristic metabolic profile. Lactate, valine, succinic acid, 3-HB, HDL, UFA, glycerol, choline, TMAO, glucose, glycine, VLDL, and Ptd may be potential biomarkers of early stage AP.

Keywords: Metabolomics, Acute pancreatitis, 1HNMR, PCA analysis, PLS-DA analysis

\section{Background}

Acute pancreatitis (AP) is a common inflammatory disease of the pancreas caused by premature activation of pancreatic enzymes. Although AP is self-limiting in $80 \%$ of patients, it can be life-threatening in the $20 \%$ who develop systemic complications, such as multiple organ failure [1]. In addition to a high mortality rate, ranging from $3-15 \%$ [2], the rate of hospitalization for AP continues to rise annually [3]. In the United States, the overall hospitalization rate has doubled over the past 20 years [4], and in the Netherlands there was a $75 \%$ increase from

\footnotetext{
* Correspondence: wftang900@gmail.com

${ }^{\dagger}$ Equal contributors

'Department of Integrative Medicine, West China Hospital, Sichuan University, Chengdu 610041, Sichuan Province, China

Full list of author information is available at the end of the article
}

1992 to 2004 [5]. A recent study suggested a 3.1\% annual increase in the incidence of AP [6] in England, and a meta-analysis from 18 European countries showed that the incidence of biliary pancreatitis has increased linearly and that the mortality rate increases with age [7]. Similar findings were reported in a study investigating the epidemiology of AP in the North Adriatic region of Croatia [8]. A population-based study from Taiwan reported that the proportion of severe cases has increased in recent years, although the overall incidence of AP remained constant [9]. Moreover, throughout the world, AP poses a heavy financial burden on the health care system. Due to unclear pathogenesis, the main treatments of AP are still supportive and symptomatic therapies, although early diagnosis and intervention may mitigate illness and reduce 
complications and length of hospitalization [10-13]. Metabolic disturbances, such as hyperglycemia and hyperlipidemia, are always present in the early stages of AP. However, the specific metabolic processes associated with this disease remain unclear.

Metabolomics is a modern approach to the study of biological samples, and can provide detailed information on the metabolic changes taking place in an organism in various pathophysiologic states. ${ }^{1} \mathrm{H}$ NMR spectroscopybased approaches are used for high-throughput research on biological samples [14-16]. Moreover, being noninvasive and providing an impartial profile of all metabolites, ${ }^{1} \mathrm{H}$ NMR-based metabolomics is widely applied in many areas related to biology and medicine, such as the exploitation of new drugs, disease diagnosis, toxicology, pharmaceutical effects, microorganism metabolomics, and identification of biomarkers [17-21]. Detection of characteristic metabolite changes in AP may increase our understanding of the pathophysiology of this disease, allow early diagnosis, and identify potential therapeutic targets. To identify potential biomarkers of AP, we analyzed the metabolic differences between rats with $\mathrm{AP}$ and healthy rats.

\section{Methods}

\section{Ethics statement}

This prospective, randomized controlled trial was performed at the ethnopharmacology laboratory of West China Hospital. The study protocol was approved by the Ethics Committee for Animal Experiments of Sichuan University. All rats were handled according to University Guidelines and the Animal Care Committee Guidelines of West China Hospital. All operations were performed under chloral hydrate anesthesia, and all efforts were made to minimize suffering.

\section{Chemicals}

Deuterium oxide $\left(\mathrm{D}_{2} \mathrm{O}, 99.9 \%\right)$ was purchased from Cambridge Isotope Laboratories, Inc (Tewksbury, MA, USA). Trimethylsilyl-propionate-2, 2, 3, 3- $\mathrm{d}_{4}$ acid, sodium salt (TSP) was purchased from Merck (Darmstadt, Germany). Sodium taurocholate was provided by Sigma Chemical (St. Louis, MO, USA). Chloral hydrate was provided by Ke Long chemical reagent works, China.

\section{Equipment}

An INOVA $600 \mathrm{MHz}$ NMR spectrometer equipped with a triple-resonance probe and a $\mathrm{z}$-axis pulsed field gradient was obtained from Varian Unity (Varian, Inc. USA), a microcentrifuge from Eppendorf MiniSpin Plus, Germany, and a two-channel micro-injection pump from Kd Scientific Company, USA.

\section{Animal model of acute pancreatitis}

Healthy male adult Sprague-Dawley rats $(224 \pm 21$ g, 200$250 \mathrm{~g} \mathrm{~b} / \mathrm{w})$, were maintained in air-conditioned animal quarters at $22 \pm 2{ }^{\circ} \mathrm{C}$ with a relative humidity of $65 \% \pm 10 \%$. They were acclimated for 1 week before the experiment with special feed and tap water. The animal experiments were carried out in the Laboratory of Ethnopharmacology at West China hospital, Sichuan University.

Fourteen 2-month-old (55-62 days old) rats obtained from the experimental animal center of Sichuan University were numbered and randomized into two groups of seven rats each, an AP group and a sham operation (SO) group. Animals were fasted for $12 \mathrm{~h}$, and given free access to tap water prior to experiments. All animals were anesthetized by intraperitoneal injection of $3 \mathrm{ml} / \mathrm{kg} 10 \%$ chloral hydrate. The hepatic duct was closed with a small bulldog clamp, and the biliopancreatic duct was cannulated through the offside of the front opening of the duodenum papilla. Rats in the AP group underwent retrograde pancreaticobiliary duct injection with $1 \mathrm{ml} / \mathrm{kg} 3.5 \%$ sodium taurocholate using an infusion pump, whereas rats in the SO group were similarly injected with $1 \mathrm{ml} / \mathrm{kg} 0.9 \%$ saline [22]. Twelve hours later, heparinized blood samples were obtained from the angular vein and centrifuged for $15 \mathrm{mi}$ nutes at $3000 \mathrm{rpm}$. Plasma samples were collected and stored at $-80^{\circ} \mathrm{C}$ until use. After the experiment, the rats were sacrificed and cremated.

\section{Sample preparation and NMR data acquisition}

Plasma samples $(150 \mu \mathrm{l})$ were mixed with $100 \mu \mathrm{l} 1 \mathrm{mg} /$ $\mathrm{ml}$ TSP in deuterium oxide $\mathrm{D}_{2} \mathrm{O}$ and $350 \mu \mathrm{l} 2 \mathrm{O}$, and centrifuged at $14000 \mathrm{rpm}$ for $10 \mathrm{~min}$. A $550 \mu \mathrm{l}$ aliquot of supernatant was transferred into $5 \mathrm{~mm}$ NMR tubes, and NMR spectra were recorded at $26^{\circ} \mathrm{C}$ using an INOVA $600 \mathrm{MHz}$ NMR spectrometer equipped with a tripleresonance probe and a z-axis pulsed field gradient. Information about micromolecular metabolites was acquired using a CPMG pulse sequence $\left[-R D-90^{\circ}-\left(r-180^{\circ}-\mathrm{r}\right)_{n}-\right.$ $\mathrm{ACQ}$ ] during a relaxation delay of $2 \mathrm{~s}$. Sixty-four free induction decays (FID) were collected into $64 \mathrm{k}$ data points with a spectral width of $8000 \mathrm{~Hz}$ and acquisition time of $4 \mathrm{~s}$. Information about macromolecular metabolites was collected by LED pulse sequence (-RD-90 $-\mathrm{G} 1-180^{\circ}-\mathrm{G} 1-$ $\left.90^{\circ}-\mathrm{T}-90^{\circ}-\mathrm{G} 1-180^{\circ}-\mathrm{G} 1-90^{\circ}-\mathrm{ACQ}\right)$ during a relaxation delay of $2 \mathrm{~s}$. Sixty-four FID were collected into $64 \mathrm{k}$ data points with a spectral width of $8000 \mathrm{~Hz}$, an acquisition time of $4 \mathrm{~s}$ and a diffusion time of $100 \mathrm{~min}$. FID was zero-filled and multiplied by an exponential line-broadening function of $1 \mathrm{~Hz}$ (for CPMG) and $3 \mathrm{~Hz}$ (for LED) prior to Fourier transformation.

\section{NMR data processing and analysis}

After Fourier transformation, the data were phased and subjected to baseline correction. For CPMG data, spectra 
in the region of $\delta$ 0.4-4.4 were subdivided into integrated regions of 0.04 PPM width. LED data were subdivided into integrated regions of 0.04 PPM width corresponding to the region of $\delta 0-6.0$, and the spectra in the $\delta 4.6-5.0$ range were excluded from data reduction. Each data point was normalized to the sum of its row to compensate for any variation in total sample volumes. The obtained data were exported to Excel and subjected to multivariate analyses as variables for the multivariate pattern recognition analysis using Soft Independent Modeling of Class Analogy (SIMCA-P) software package (v10.04, Umetrics, Umeå, Sweden). Principal components analysis (PCA) was performed after data were pretreated by mean centering and Pareto scaling. For samples with obvious internal individual differences, partial least squares (PLS) and discriminant analysis (DA) were conducted after data processing based on orthogonal signal correction (OSC). These two approaches were performed to differentiate between sample groups. Score plots were used to visualize the separation of the groups, while the loading plots were used to determine which spectral variables significantly contributed to the separation of the samples on the score plots.

\section{Results}

1H-NMR spectra (CPMG) and pattern recognition analysis of plasma samples

A comparison of the $1 \mathrm{H}-\mathrm{NMR}$ spectra of plasma samples taken from rats in the AP and SO groups showed obvious differences in the levels of small molecule metabolites (Figure 1). To identify these differences in metabolic profiles, the $1 \mathrm{H}-\mathrm{NMR}$ spectra were segmented and subjected to PCA. The scores plot showed a cluster distribution of the two groups without distinct separation, with the point AP10 located outside the confidence interval (Figure 2a). The clusters became more obviously distributed after excluding the AP10 point (Figure $2 \mathrm{~b}$ ). With the loading plot (Figure 2d), a sequence of metabolic alterations was detected in plasma samples from rats with AP: (1) increased levels of lactate, valine, succinic acid, and 3hydroxybutyric acid (3-HB); and (2) decreased levels of glycerol, choline, trimethylamine oxide (TMAO), glucose, and glycine (Table 1). These altered metabolites may be potential biomarkers of AP.

\section{H-NMR spectra (LED) and pattern recognition analysis of plasma samples}

Similar to the analysis of small molecule metabolites, the levels of macromolecule lipid metabolites differed markedly between the AP and SO groups, as shown by the 1H-NMR (LED) spectra of their plasma samples (Figure 3). To illustrate the differences in metabolic profiles required OSC-PLS (ctr) analysis (Figure 4a,b) was conducted because PCA analysis was not able to detect differences between these two groups (Figure 4c,d). The score plot showed distinct separation between the two groups (Figure 4a), with the loading plot (Figure 4b) showing increases in high density lipoproteins (HDL, 0.86 ) and unsaturated fatty acids (UFA, 2.78, 5.3), and decreases in very low density lipoprotein [(VLDL, 1.34), (VLDL/LDL, 1.3)] and phosphatidylcholine (Ptd, 2.78) (Table 2). Therefore, HDL, UFA, VLDL, and Ptd may be potential biomarkers of AP.

\section{Discussion}

By analyzing the plasma metabolites of rats with experimental AP using the 1H-NMR-based metabolomic approach, we obtained a series of finger-prints containing information on plasma metabolites. Except for one point, the separation between the two groups was distinct, as shown in the score plot of PCA models of the CPMG data for small molecules. Similarly, we observed a separation of metabolites between the two groups in the score plot of

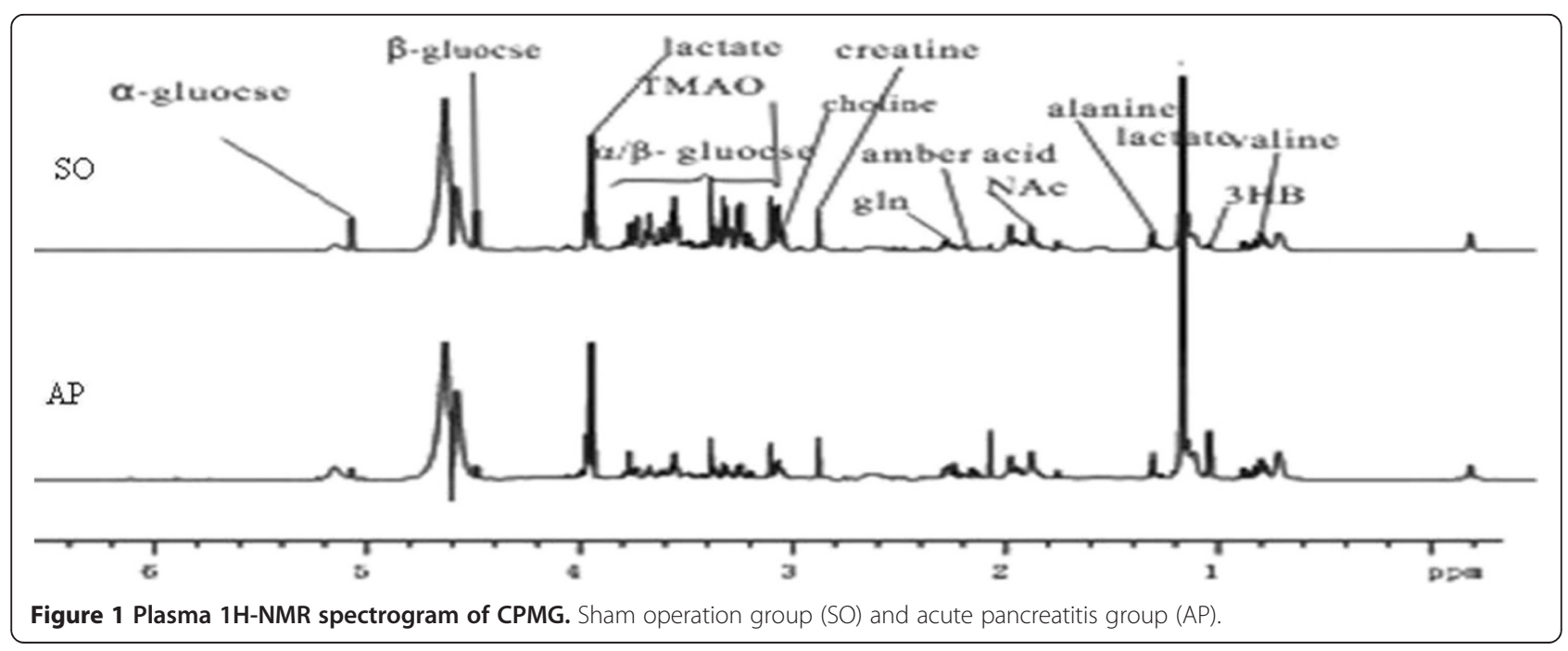




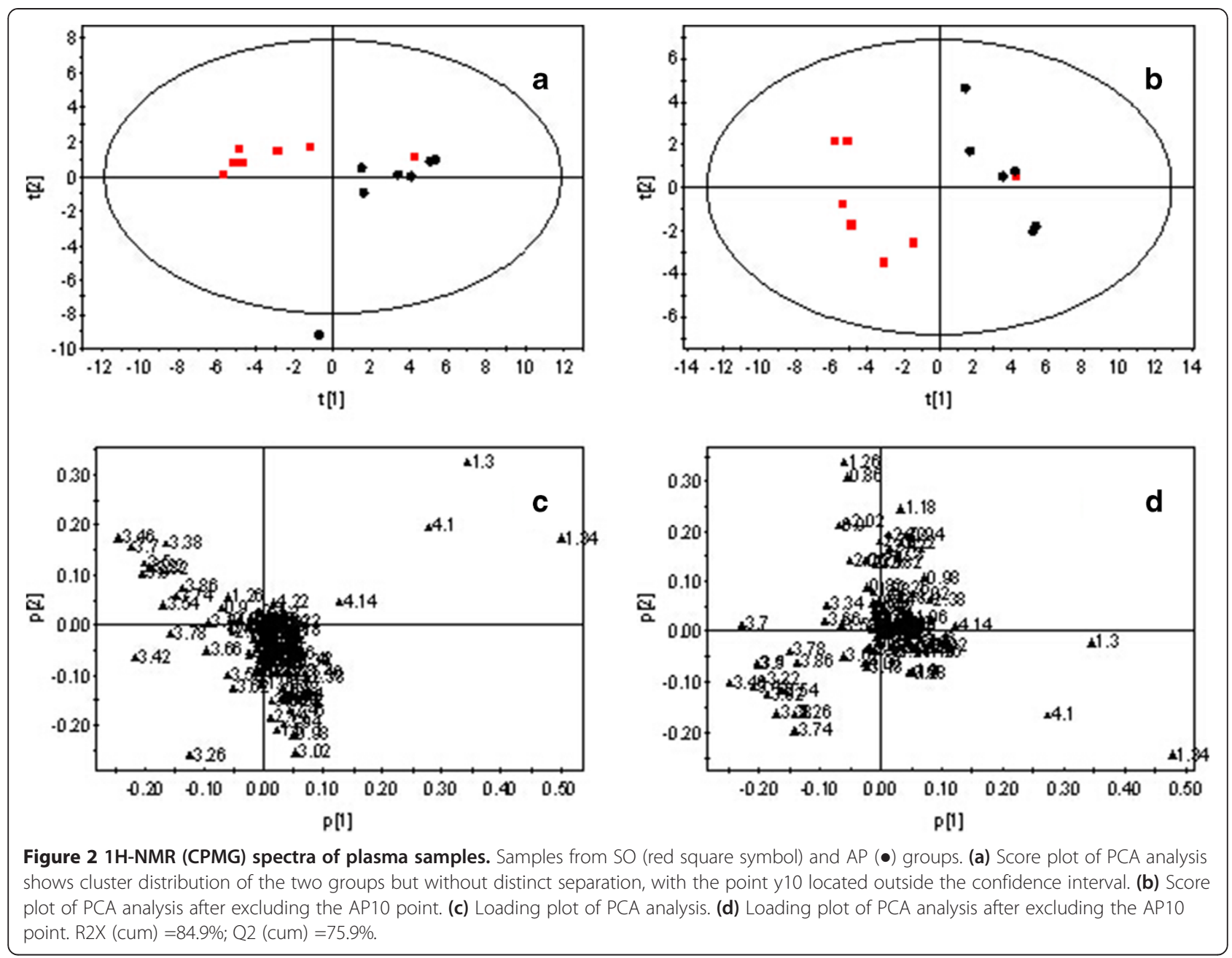

Table 1 Levels of small molecule metabolites in the AP and SO groups

\begin{tabular}{lll}
\hline Chemical shift (ppm) & Name of compound & $\begin{array}{l}\text { Acute pancreatitis } \\
\text { group (AP) }\end{array}$ \\
\hline $0.98,1.02$ & Valine & $\uparrow$ \\
$1.3,1.34,4.1$ & Lactate & $\uparrow$ \\
$3.58,3.66$ & Glycerol & $\downarrow$ \\
2.38 & succinic acid & $\uparrow$ \\
1.18 & $3-$ HB & $\uparrow$ \\
3.22 & Choline & $\downarrow$ \\
3.26 & TMAO & $\downarrow$ \\
$3 \sim 4$ & Glucose & $\downarrow$ \\
3.54 & Glycine & $\downarrow$ \\
\hline
\end{tabular}

$\uparrow$ : elevated; $\downarrow$ : reduced; 3-HB: 3-hydroxybutyric acid; TMAO: trimethylamine oxide. All of the results were compared with sham operation (SO) group.
PLS-DA models for macromolecules, although the two groups were mixed by PCA.

The plasma $1 \mathrm{H}-\mathrm{NMR}$ spectra of CPMG data for small molecules showed a difference between the two groups. The score plot with PCA of 1H-NMR (CPMG) spectra of plasma samples showed that most of the metabolites in the AP group could be separated from those in the SO group, indicating a significant difference in metabolomics between AP and control rats. The score plot with PCA of 1H-NMR (LED) spectra from the two groups showed a cross distribution. The cluster distribution of the PLS-DA could distinguish between the two groups, thus illustrating their metabolomic differences.

This study also showed differences in plasma metabolite levels between the two groups. Rats with AP had higher levels of lactate, valine, succinic acid, 3hydroxybutyrate (3-HB), HDL, and UFA, and lower levels of glycerol, choline, TMAO, glucose, glycine, VLDL, and phosphatidylcholine, than the control group. 

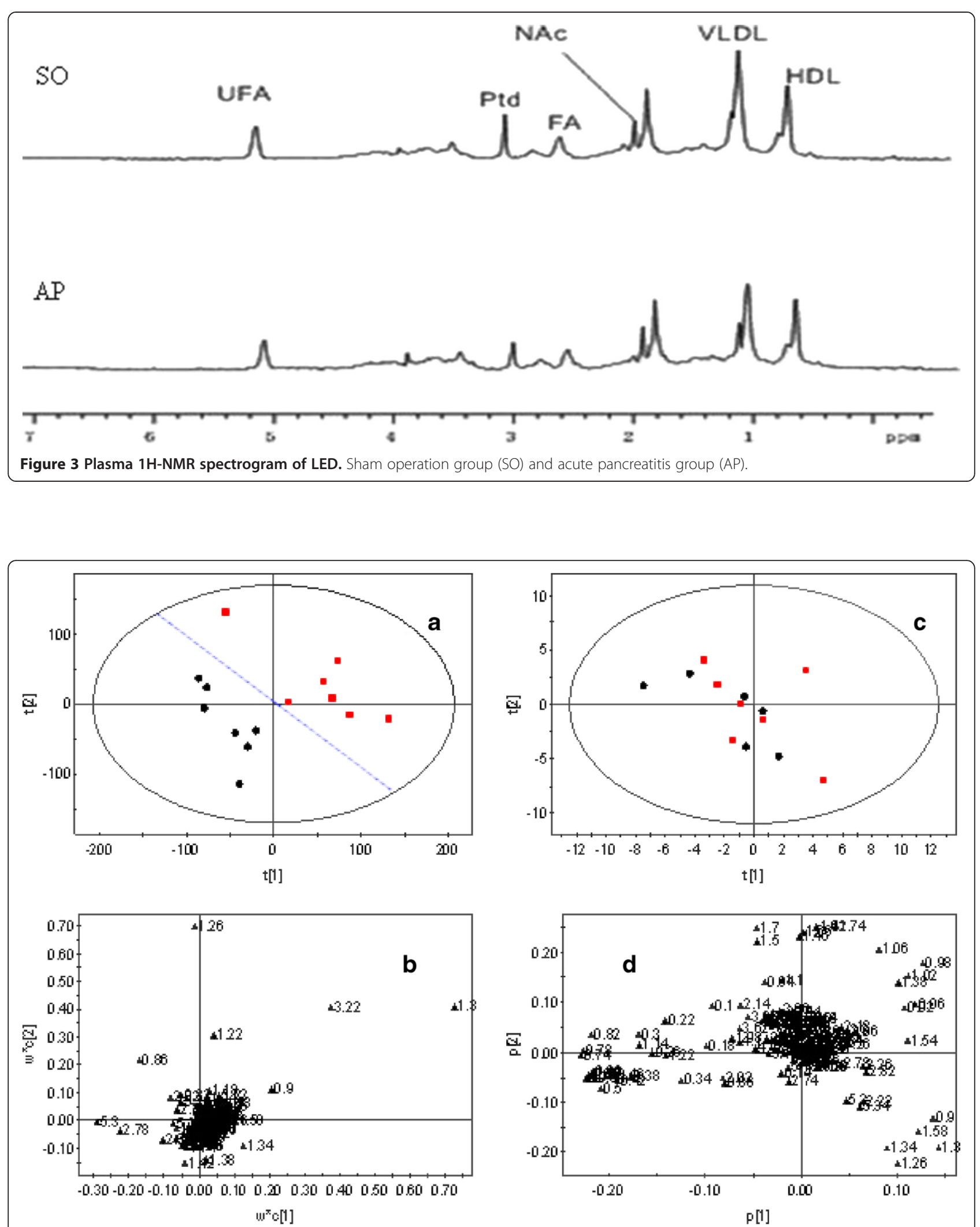

Figure 4 1H-NMR (LED) spectra of plasma samples. Samples from SO (red square symbol) and AP (•) groups. (a) Score plot of OSC-PLS-DA. (b) Loading plot of OSC-PLS-DA. R2X (cum) =70.2\%; R2Y (cum) =87.1\%; Q2 (cum) =72.4\%. (c) Score plot of PCA analysis. (d) Loading plot of PCA analysis. 
Table 2 Levels of macromolecular lipid metabolites in the AP and SO groups

\begin{tabular}{lll}
\hline Chemical shift (ppm) & Name of compound & $\begin{array}{l}\text { Acute pancreatitis } \\
\text { group (AP) }\end{array}$ \\
\hline 0.86 & HDL & $\uparrow$ \\
1.22 & LDL & $-/ \downarrow$ \\
1.3 & VLDL/LDL & $\downarrow$ \\
0.9 & HDL/LDL & $\downarrow$ \\
1.34 & VLDL & $\downarrow$ \\
2.78 & UFA & $\uparrow$ \\
3.22 & Ptd & $\downarrow$ \\
5.3 & UFA & $\uparrow$ \\
\hline
\end{tabular}

$\uparrow$ : elevated; $\downarrow$ : reduced; -: similar; HDL: high density lipoprotein; LDL: low density lipoprotein; VLDL: very low density lipoprotein; FA: saturated fatty acid; Ptd: phosphatidylcholine; UFA: unsaturated fatty acid. All of the results were compared with the sham operation (SO) group.

Because of acute systemic inflammation response syndrome, AP always triggers a hypercatabolic state, resulting in increased energy requirements and protein catabolism [23]. Our results indicate that multiple metabolic pathways were mobilized, resulting in changes in metabolite concentrations. Lactate is mainly generated by anaerobic glycolysis and is not associated with the primary energy supply pathway under normal conditions. During an intense workout or under conditions of hypoxia, an insufficient oxygen supply can reduce Krebs cycle reactions, produce less ATP, and enhance glycolysis, resulting in decreased removal ability of the liver and kidneys and ultimately to the accumulation of lactate in plasma $[24,25]$. The increased levels of lactate and succinic acid in the AP group were likely because of anaerobic glycolysis, which was enhanced by the increased energy consumption and oxygen deficit during early stages of AP [26]. Since lactate concentration is influenced by oxygen supply and fluid resuscitation, this elevation was not detected in urine samples from patients with pancreatitis [27]. As an aerobic process, gluconeogenesis was inhibited by an inadequate oxygen supply, which may be partly responsible for the decrease in glucose level. Further, insulin released from injured acinar cells also contributed to decreased glucose levels. TMAO, the product of dietary choline and L-carnitine [28] , although mainly regarded as a biomarker of renal function [29], may participate in energy production as an external electron acceptor in an hypoxic environment[30]. Moreover, decreased TMAO has been reported to accompany a chemiosmotic mechanism of energy conversion [31]. The decreased TMAO we observed may indicate renal damage caused by AP as well as energy conversion under conditions of relative oxygen deficit. Similar changes in TMAO have been observed in serum samples of patients with pancreatitis [32]. In contrast, 3-HB, a ketone body synthesized in the liver and used as an energy source by the brain under conditions of hypoglycemia or enhanced demand for gluconeogenesis [33], was not increased in serum samples of patients with pancreatitis [32]. Inflammatory responses in AP progressively damage pancreatic acinar cells and impair mitochondrial respiratory capacity, limiting ATP production [34]. In addition, increased energy consumption mobilizes FA to the periphery as an energy supply, reducing FA and increasing 3-HB. This results in increased HDL level at the periphery, where it is required to carry cholesterol and triacylglycerol. Serum VLDL transports triacylglycerol into tissue for energy metabolism, and phosphatidylcholine moves into tissue to preserve cell function, leading to elevated serum HDL levels and decreased LDL, VLDL, and phosphatidylcholine levels. UFA, which is important in maintaining the relative liquidity of cell membranes and normal cell function, is released into circulation under stress, damaging the stability of cell membranes [35]. Moreover, glutaminate production is increased to protect against tissue injury, and alanine accumulates to inhibit the proteolysis of skeletal muscle proteins, resulting in increased serum levels of glutaminate and alanine.

One of the limitations of this study was that we only investigated changes in plasma concentrations of metabolic factors. Further studies investigating urine and pancreatic tissue samples are needed to confirm our findings. Moreover, insufficient plasma was available for assay of all factors, and this study was based on the 1HNMR approach, which in itself is limited by the incomplete data obtained. The combined use of LC-MS or GC-MS is needed to validate our results.

\section{Conclusion}

We found that the characteristic metabolomic profile differed in AP and SO rats. Lactate, valine, succinic acid, 3HB, HDL, UFA, glycerol, choline, TMAO, glucose, glycine, VLDL, and Ptd may be regarded as potential biomarkers of early stage AP. These findings may provide deeper insight into the pathophysiology and metabolic state of AP and may facilitate early intervention in patients with this disease. The 1HNMR-based metabolomic approach was capable of distinguishing between plasma samples of AP rats and SO controls, providing a new and non-invasive methodology for the study of AP.

\section{Abbreviations}

AP: Acute pancreatitis; CPMG: Carr-Purcell-Meiboom-Gill; DA: Discriminant analysis; FID: Free induction decays; LED: Longitudinal Eddy-delay; NMR: Nuclear magnetic resonance; OSC: Orthogonal signal correction; PLS: Partial least squares; PCA: Principal components analysis; SIMCA-P: Soft independent modeling of class analogy; LC-MS: Liquid chromatography-mass spectrometry; GC-MS: Gas chromatography-mass spectrometry; HDL/LDL/NLDL: High/low/ very low density lipoprotein; FAVUFA: Saturated/unsaturated fatty acid; TMAO: Trimethylamine N-oxide; NAC: N-acetyl glycoprotein.

\section{Competing interests}

The authors declare that they have no competing interests. 


\section{Authors' contributions}

WFT, study concept and design; YXL, XHP and MHW, acquisition of data; analysis and interpretation of data; $J \mathrm{~L}$ and $\mathrm{XLZ}$, drafting of the manuscript WFT, critical revision of the manuscript for important intellectual content; YXL and SFZ, statistical analysis; WFT, obtained funding; YLL and $H G$, material support; WFT, study supervision. All authors read and approved the final manuscript.

\section{Acknowledgments}

We gratefully acknowledge Qi Zhang and Juan Liu of the NMR Laboratory of the National Center of Biomedical Analysis, Academy of Military Medical Sciences, for performing metabolomic analysis. This study received financial support from the National Natural Science Foundation of China (Grant number: 30672588,81374042$)$. The funders had no role in study design, data collection and analysis, decision to publish, or preparation of the manuscript.

\section{Author details}

'Department of Integrative Medicine, West China Hospital, Sichuan University, Chengdu 610041, Sichuan Province, China. ${ }^{2}$ China Tibetology Research Center, 100101 Beijing, China.

Received: 26 November 2013 Accepted: 19 June 2014 Published: 30 June 2014

\section{References}

1. Frossard JL, Steer ML, Pastor CM: Acute pancreatitis. Lancet 2008, 371(9607):143-152

2. van Santvoort HC, Bakker OJ, Bollen TL, Besselink MG, Ahmed Ali U, Schrijver AM, Boermeester MA, van Goor H, Dejong $\mathrm{CH}$, van Eijck $\mathrm{CH}$, van Ramshorst B, Schaapherder AF, van der Harst E, Hofker S, Nieuwenhuijs VB, Brink MA, Kruyt PM, Manusama ER, van der Schelling GP, Karsten T, Hesselink EJ, van Laarhoven CJ, Rosman C, Bosscha K, de Wit RJ, Houdijk AP, Cuesta MA, Wahab PJ, Gooszen HG, Dutch Pancreatitis Study Group: A conservative and minimally invasive approach to necrotizing pancreatitis improves outcome. Gastroenterology 2011, 141:1254-1263.

3. Fagenholz PJ, Castillo CF, Harris NS, Pelletier AJ, Camargo CA Jr: Increasing United States hospital admissions for acute pancreatitis, 1988-2003. Ann Epidemiol 2007, 17:491-497.

4. Lowenfels AB, Maisonneuve P, Sullivan T: The changing character of acute pancreatitis: epidemiology, etiology, and prognosis. Curr Gastroenterol Rep 2009, 11(2):97-103.

5. Spanier BW, Dijkgraaf MG, Bruno MJ: Trends and forecasts of hospital admissions for acute and chronic pancreatitis in the Netherlands. Eur J Gastroenterol Hepatol 2008, 20(7):653-658.

6. Roberts SE, Williams JG, Meddings D, Goldacre MJ: Incidence and case fatality for acute pancreatitis in England: geographical variation, social deprivation, alcohol consumption and aetiology-a record linkage study. Aliment Pharmacol Ther 2008, 28(7):931-941.

7. Yadav D, Lowenfels AB: Trends in the epidemiology of the first attack of acute pancreatitis: a systematic review. Pancreas 2006, 33:323-330.

8. Stimac D, Mikolasevic I, Krznaric-Zrnic I, Radic M, Milic S: Epidemiology of acute pancreatitis in the north adriatic region of Croatia during the last ten years. Gastroenterol Res Pract 2013. Epub 2013 Feb 14.

9. Shen HN, Lu CL, Li CY: Epidemiology of first-attack acute pancreatitis in Taiwan from 2000 through 2009: a nationwide population-based study. Pancreas 2012, 41(5):696-702.

10. Wu BU, Conwell DL: Acute pancreatitis part I: approach to early management. Clin Gastroenterol Hepatol 2010, 8:410-416.

11. Pandol SJ, Saluja AK, Imrie CW, Banks PA: Acute pancreatitis: bench to the bedside. Gastroenterology 2007, 132(3):1127-1151.

12. Warndorf MG, Kurtzman JT, Bartel MJ, Cox M, Mackenzie T, Robinson S, Burchard PR, Gordon SR, Gardner TB: Early fluid resuscitation reduces morbidity among patients with acute pancreatitis. Clin Gastroenterol Hepatol 2011, 9(8):705-709.

13. Fisher JM, Gardner TB: The "golden hours" of management in acute pancreatitis. Am J Gastroenterol 2012, 107(8):1146-1150.

14. Bollard ME, Stanley EG, Lindon JC, Nicholson JK, Holmes E: NMR-based metabonomic approaches for evaluating physiological influences on biofluid composition. NMR Biomed 2005, 18:143-162.
15. Serkova NJ, Spratlin JL, Eckhardt SG: NMR-based metabolomics: translational application and treatment of cancer. Curr Opin Mol Ther 2007, 9:572-585.

16. Madsen R, Lundstedt T, Trygg J: Chemometrics in metabolomics-a review in human disease diagnosis. Anal Chim Acta 2010, 659(1-2):23-33.

17. Desmoulin F, Galinier M, Trouillet C, Berry M, Delmas C, Turkieh A, Massabuau P, Taegtmeyer H, Smih F, Rouet P: Metabonomics analysis of plasma reveals the lactate to cholesterol ratio as an independent prognostic factor of short-term mortality in acute heart failure. PLoS One 2013, 8(4):e60737.

18. Guan M, Xie L, Diao C, Wang N, Hu W, Zheng Y, Jin L, Yan Z, Gao H: Systemic perturbations of key metabolites in diabetic rats during the evolution of diabetes studied by urine metabonomics. PLoS One 2013, 8(4):e60409.

19. Morin PJ, Ferguson D, Leblanc LM, Hébert MJ, Paré AF, Jean-François J, Surette ME, Touaibia M, Cuperlovic-Culf M: NMR metabolomics analysis of the effects of 5-lipoxygenase inhibitors on metabolism in glioblastomas. $J$ Proteome Res 2013. Epub ahead of print.

20. Kobayashi T, Nishiumi S, Ikeda A, Yoshie T, Sakai A, Matsubara A, Izumi Y, Tsumura H, Tsuda M, Nishisaki H, Hayashi N, Kawano S, Fujiwara Y, Minami H, Takenawa T, Azuma T, Yoshida M: A novel serum metabolomics-based diagnostic approach to pancreatic cancer. Cancer Epidemiol Biomarkers Prev 2013, 22(4):571-579.

21. Sakai A, Nishiumi S, Shiomi Y, Kobayashi T, Izumi Y, Kutsumi H, Hayakumo T, Azuma T, Yoshida M: Metabolomic analysis to discover candidate therapeutic agents against acute pancreatitis. Arch Biochem Biophys 2012, 522(2):107-120.

22. Gong HL, Tang WF, Yu Q, Xiang J, Xia Q, Chen GY, Huang X, Liang MZ: Effect of severe acute pancreatitis on pharmacokinetics of Da-Cheng-Qi Decoction components. World J Gastroenterol 2009, 15(47):5992-5999.

23. Working Party of the British Society of Gastroenterology: UK guidelines for the management of acute pancreatitis. Gut 2005, 54(supplement 3):iii1-iii9.

24. He G, Jiang Y, Zhang B, Wu G: The effect of HIF-1a on glucose metabolism, growth and apoptosis of pancreatic cancerous cells. Asia Pac J Clin Nutr 2014, 23(1):174-180.

25. Bartrons R, Caro J: Hypoxia, glucose metabolism and the Warburg's effect. J Bioenerg Biomembr 2007, 39:223-229.

26. Singh $A$, Chen $M, L i T$, Yang XL, Li JZ, Gong JP: Parenteral nutrition combined with enteral nutrition for severe acute pancreatitis. ISRN Gastroenterol 2012, 2012:791383.

27. Lusczek ER, Paulo JA, Saltzman JR, Kadiyala V, Banks PA, Beilman G, Conwell DL: Urinary $1 \mathrm{H}-\mathrm{NMR}$ metabolomics can distinguish pancreatitis patients from healthy controls. JOP 2013, 14(2):161-170.

28. Zhang AQ, Mitchell SC, Smith RL: Dietary precursors of trimethylamine in man: a pilot study. Food Chem Toxicol 1999, 37:515-520.

29. Wang Y, Bollard ME, Nicholson JK, Holmes E: Exploration of the direct metabolic effects of mercury II chloride on the kidney of Sprague-Dawley rats using high-resolution magic angle spinning $1 \mathrm{H}$ NMR spectroscopy of intact tissue and pattern recognition. J Pharm Biomed Anal 2006, 40(2):375-381.

30. Oren A: Formation and breakdown of glycine betaine and trimethylamine in hypersaline environments. Antonie Van Leeuwenhoek 1990, 58(4):291-298.

31. Stenberg E, Ringø E, Strøm AR: Trimethylamine oxide respiration of Alteromonas putrefaciens NCMB 1735: Na +-stimulated anaerobic transport in cells and membrane vesicles. Appl Environ Microbiol 1984, 47(5):1090-1095.

32. Ouyang D: Metabolomic characterization of human pancreatitis by ${ }^{1}$ H-NMR spectroscopy. Hepatogastroenterology 2012, 59(119):2314-2317.

33. Diaz SO, Pinto J, Graça G, Duarte IF, Barros AS, Galhano E, Pita C, Almeida Mdo C, Goodfellow BJ, Carreira IM, Gil AM: Metabolic biomarkers of prenatal disorders: an exploratory NMR metabonomics study of second trimester maternal urine and blood plasma. J Proteome Res 2011, 10(8):3732-3742.

34. Maléth J, Rakonczay Z Jr, Venglovecz V, Dolman NJ, Hegyi P: Central role of mitochondrial injury in the pathogenesis of acute pancreatitis. Acta Physiol (Oxf) 2013, 207(2):226-235.

35. Hac-Wydro K1, Wydro P: The influence of fatty acids on model cholesterol/ phospholipid membranes. Chem Phys Lipids 2007, 150(1):66-81.

doi:10.1186/1471-230X-14-115

Cite this article as: Li et al: 1HNMR-based metabolomic profile of rats with experimental acute pancreatitis. BMC Gastroenterology 2014 14:115 